This tensor vanishes identically for a projective space of two dimensions. For spaces of three or more dimensions, the Weyl projective curvature tensor vanishes if and only if the given affine space is projectively equivalent to a flat space.

Although the book seems to lack geometrical motivation, the material is presented in a very understandable fashion. The ideas and concepts are given very concisely and thus a wide range of subjects is covered. This book is a very worthwhile introduction to the subject for a beginning student of the absolute differential calculus and its applications.

JoHN DeCrcco

\title{
Brief Mention
}

The hodograph method in gas dynamics. By A. G. Ghaffari. Teheran, Taban, 1950. 129 pp., 19 figs.

This book gives an introductory account of one of the most interesting methods in gas dynamics, the hodograph method. Particular emphasis has been given to approximate methods (Chapters IV, V, VII), including some of the author's own work. Chaplygin's method and its current developments are discussed briefly in Chapter III, while Chapter VI gives a rather detailed account of Bergman's method. A clear presentation of the standard material in gas dynamics is given in Chapters I, II, III, and VIII. The book contains a preface by Professor G. Temple which concludes with the statement: "This book can be confidently recommended to students of aerodynamics who desire an elementary survey of the theory and use of the Hodograph method."

$$
\text { C. C. LIN }
$$

Gelöste und ungelöste mathematische Probleme aus alter und neuer Zeit.

By Heinrich Tietze. Munich, Biederstein, 1949; vol. 1, 20+256 pp.; vol. 2, 4+305 pp.

This is one of the deepest and at the same time the most charming of the popular books on mathematics that I have ever seen. In two moderate sized volumes Tietze presents fourteen lectures, on topics as diverse as digital representations of integers, distribution of primes, properties of geodesics on surfaces, dimension theory, the regular 17-gon, solution of equations by radicals, and the concept of infinity. The lectures are addressed to a lay audience. A mathematically untrained reader of the book (assuming that he has no difficulty with Tietze's picturesque German) can get a lot of understanding and 